\title{
PROPERTIES OF RELATIVELY FREE INVERSE SEMIGROUPS
}

BY

\author{
N. R. REILLY AND P. G. TROTTER
}

\begin{abstract}
The objective of this paper is to study structural properties of relatively free inverse semigroups in varieties of inverse semigroups. It is shown, for example, that if $S$ is combinatorial (i.e., $\not$ is trivial), completely semisimple (i.e., every principal factor is a Brandt semigroup or, equivalently, $S$ does not contain a copy of the bicyclic semigroup) or $E$-unitary (i.e., $E(S)$ is the kernel of the minimum group congruence) then the relatively free inverse semigroup $F \mathcal{V}_{X}$ on the set $X$ in the variety $\mathcal{V}$ generated by $S$ is also combinatorial, completely semisimple or $E$-unitary, respectively.

If $S$ is a fundamental (i.e., the only congruence contained in $H$ is the identity congruence) and $|X| \geq \mathcal{\aleph}_{0}$, then $F \mathcal{V}_{X}$ is also fundamental. $F \mathcal{V}_{X}$ may not be fundamental if $|X|<\aleph_{0}$. It is also shown that for any variety of groups $U$ and for $|X| \geq \aleph_{0}$, there exists a variety of inverse semigroups $\mathcal{V}$ which is minimal with respect to the properties (i) $F \mathcal{V}_{X}$ is fundamental and (ii) $\mathcal{V} \cap \mathcal{G}=\mathcal{U}$, where $\mathcal{G}$ is the variety of groups.

In the main result of the paper it is shown that there exists a variety $\mathcal{V}$ for which $F V_{X}$ is not completely semisimple, thereby refuting a long standing conjecture.
\end{abstract}

1. Summary. In general, the relatively free objects in any variety of algebras are important in the study of that variety and this has been true, in particular, in the study of inverse semigroups. The first good description of the free inverse semigroup on one generator was given by Gluskin [2], while the first good description of the free inverse semigroup on an arbitrary set was given by Scheiblich $[\mathbf{1 2}]$. For a survey of these and related results, see Petrich [6] and Reilly [10].

The structures of the free group, the free inverse semigroup and free semilattice of groups are well known. However, with the one exception of the work on the relatively free objects in varieties generated by $E$-unitary inverse semigroups by Pastijn [4] and Petrich and Reilly [7], relatively little has been said regarding the structure of the relatively free objects in other varieties of inverse semigroups or with regard to other properties than $E$-unitary.

Since it would seem to be beyond the present state of knowledge to give explicit structure theorems for nontrivial relatively free inverse semigroups other than those considered by Gluskin and Scheiblich, we continue the investigation of the structure of relatively free inverse semigroups in the spirit of Pastijn [4] and Petrich and Reilly [7].

$\S 2$ is devoted to background information. In $\S 3$, it is shown that certain structural properties of an inverse semigroup $S$ will be inherited by the relatively free objects $F \mathcal{V}_{X}$, in the variety $\mathcal{V}$ generated by $X$. If $S$ is combinatorial or completely

Received by the editors March 5, 1985 .

1980 Mathematics Subject Classification. Primary 20M07; Secondary 20M05, 20M10. 
semisimple, then so is $F \mathcal{V}_{X}$, while if $|X| \geq \aleph_{0}$ and $S$ is fundamental, then so is $F \mathcal{V}_{X}$

If $\mathcal{V}$ and $\mathcal{W}$ are varieties such that $\mathcal{V} \subseteq \mathcal{W}$ and $\operatorname{ker} \rho \mathcal{V}=\operatorname{ker} \rho_{\mathcal{W}}$ (where $\rho \mathcal{V}$ denotes the fully invariant congruence on the free inverse semigroup on $\aleph_{0}$ generators that defines $\mathcal{V}$ ), then it is shown that $F \mathcal{W}_{X}$ will be $E$-unitary, combinatorial or fundamental if $F \mathcal{V}_{X}$ is and that $F \mathcal{W}_{X}$ will be completely semisimple if $F \mathcal{V}_{X}$ is both completely semisimple and combinatorial.

It has long been conjectured that all relatively free inverse semigroups might be completely semisimple. $\S 4$ is devoted to showing that this is not the case.

In $\S 5$ it is shown that if $\mathcal{G}$ is the variety of all groups and $\mathcal{U}$ is a variety of groups then there exists a variety $\mathcal{V}$ of inverse semigroups which is minimal with respect to the properties that (i) $\mathcal{V} \cap \mathcal{G}=\mathcal{U}$ and (ii) for $|X| \geq \aleph_{0}, F \mathcal{V}_{X}$ is fundamental.

In $\S 6$, it is shown that there is a countably infinite family of varieties with relatively free objects that are combinatorial, completely semisimple and $E$-unitary while, in $\S 7$, it is shown that there is a countably infinite family of varieties with relatively free objects that are fundamental, completely semisimple and $E$-unitary but not combinatorial.

2. Notation and terminology. We shall adopt the notation and terminology to be found in Howie $[\mathbf{3}]$ or Petrich [6], to which books the reader is referred for basic information on inverse semigroups.

We shall adopt the following notation for certain specific varieties:

I - the variety of all inverse semigroups,

$C$ - the variety of inverse semigroups generated by the bicyclic semigroup $C$,

$\mathcal{G}$-the variety of all groups,

$A G$ - the variety of all abelian groups,

$\mathcal{V}(S)$ - the variety of inverse semigroups generated by $S$.

For any set $X$, we will denote by $F_{X}$ the free semigroup on $X$ and, for any variety of inverse semigroups $\mathcal{V}$, we will denote by $F \mathcal{V}_{X}$ the relatively free inverse semigroup in $\mathcal{V}$ on $X$. If we wish to focus on the cardinality of $X$, we will write $F_{n}(\mathcal{V})$ (respectively, $F_{\aleph_{0}}(\mathcal{V})$ ) for $F \mathcal{V}_{X}$ with $|X|=n$ (respectively, $\aleph_{0}$ ).

If $|X|=\aleph_{0}$, then each variety of inverse semigroups, $\mathcal{V}$, is completely determined by a fully invariant congruence on $F I_{X}$. We shall denote this congruence by $\rho \mathcal{V}$.

We shall want to take advantage of two ways of describing the free inverse semigroup on a set $X$.

Let $X^{-1}$ denote a set disjoint from $X$ and in one-to-one correspondence with $X$ via $x \leftrightarrow x^{-1}$. Now extend this to a unary operation $a \rightarrow a^{-1}$ on the free semigroup $F_{X \cup X^{-1}}$ by defining

$$
\left(x^{-1}\right)^{-1}=x, \quad(a b)^{-1}=b^{-1} a^{-1}
$$

for all $x \in X, a, b \in F_{X \cup X^{-1}}$. Let $\rho$ be the smallest congruence on $F_{X \cup X^{-1}}$ such that

$$
\left(a, a a^{-1} a\right) \in \rho, \quad\left(a a^{-1} b b^{-1}, b b^{-1} a a^{-1}\right) \in \rho
$$

for all $a, b \in F_{X \cup X^{-1}}$. Then $F_{X \cup X^{-1}} / \rho$ is the free inverse semigroup $F I_{X}$ on $X$. Since our focus is on $F I_{X}$ and not $F_{X \cup X^{-1}}$, we will suppress the congruence $\rho$. Then each element of $F I_{X}$ can be written as a word which is a product of elements of the form $x$ and $y^{-1}, x, y \in X$. 
There is a natural homomorphism of $F I_{X}$ onto the free group $F \mathcal{G}_{X}$ on $X$, where $F \mathcal{G}_{X}$ is considered as the set of all reduced words on $X \cup X^{-1}$. For any word $w \in F I_{X}$, let $\bar{w}$ denote the "reduced form" of $w$ obtained by deleting successively all adjacent pairs of the form $a a^{-1}$. Then $w \rightarrow \bar{w}$ is an epimorphism of $F I_{X}$ onto $F \mathcal{G}_{X}$.

Every word $w$ in $F I_{X}$ can be written in the form $w=a_{1} a_{1}^{-1} a_{2} a_{2}^{-1} \cdots a_{m} a_{m}^{-1} a$ where the $a$ and $a_{i}$ contain no successive terms of the form $b b^{-1}$. Thus $a$, considered as a word in $F G_{X}$, is reduced as written so that $\bar{w}=a$ and we can write $w$ in the canonical form

$$
w=a_{1} a_{1}^{-1} \cdots a_{m} a_{m}^{-1} \bar{w} .
$$

We also wish to take advantage of Scheiblich's construction of $F I_{X}$. Let 1 denote the identity of $F g_{X}$. We will say that a subset $A$ of $F g_{X}$ is convex if $A$ contains all initial segments, including 1 , of all words (in reduced form) in $A$. Let $\mathcal{Y}$ denote the set of all finite convex subsets of $F G_{X}$ containing 1 and at least one other element. Let

$$
F=\left\{(A, g) \in \mathcal{Y} \times F \mathcal{G}_{X}: g \in A\right\}
$$

and define a product in $F$ by

$$
(A, g)(B, h)=(A \cup g B, g h) .
$$

Then $F$ is the free inverse semigroup on $X$ (with respect to the embedding $x \rightarrow$ $(\{1, x\}, x))$. The isomorphism between $F I_{X}$ considered as words on the alphabet $X \cup X^{-1}$ and Scheiblich's description is given as follows. For any word $w \in F I_{X}$, let

$$
A(w)=\{\bar{u}: u \text { is an initial segment of } w\} .
$$

If $w$ is written in the canonical form (1), then

$$
A(w)=\left(\bigcup A\left(a_{i}\right)\right) \cup A(\bar{w})
$$

and the mapping

$$
w \rightarrow(A(w), \bar{w})
$$

is an isomorphism of $F I_{X}$ onto $F$.

Henceforth, we will pass between these two descriptions of the free inverse semigroup on $X$, as convenient.

With regard to computations within Scheiblich's description of $F I_{X}$, the following observations will be useful: for any $A \in \mathcal{Y}, g \in A, u, v \in F I_{X}$,

$$
\begin{gathered}
(A, g)^{-1}=\left(g^{-1} A, g^{-1}\right), \\
A(u v)=A(u) \cup \bar{u} A(v), \quad A\left(u^{-1}\right)=\bar{u}^{-1} A(u) .
\end{gathered}
$$

Also, for any $w \in F I_{X}$, written as a word in $X \cup X^{-1}$, the content $c(w)$ of $w$ is the set of elements of $X$ appearing in $w$. $S$.

For any inverse semigroup $S$, let $E(S)$ denote the semilattice of idempotents of

For any congruence $\rho$ on an inverse semigroup $S$,

$$
\begin{aligned}
\operatorname{ker} \rho & =\left\{a \in S: a \rho a a^{-1}\right\}=\left\{a \in S:(a \rho)^{2}=a \rho\right\}, \\
\operatorname{tr} \rho & =\rho \cap(E(S) \times E(S)) .
\end{aligned}
$$


Any congruence $\rho$ on an inverse semigroup $S$ is completely determined by ker $\rho$, the kernel of $\rho$, and $\operatorname{tr} \rho$, the trace of $\rho$ (see Petrich [6]). We say that $\rho$ is idempotent pure if and only if $\operatorname{ker} \rho=E(S)$ and that $\rho$ is idempotent separating if and only if $\operatorname{tr} \rho=\iota_{E(S)}$. Equivalently, $\rho$ is idempotent separating if and only if $\rho \subseteq \mathcal{H}$.

On any inverse semigroup $S$, there is a minimum congruence $\sigma=\sigma_{S}$ such that $S / \sigma$ is a group. If $\operatorname{ker} \sigma=E(S)$, then $S$ is said to be $E$-unitary (over the group $G=$ $S / \sigma)$. There is also a maximum congruence $\mu=\mu_{S}$ (or, $\mu(S)$ ) on $S$ such that $\mu \subseteq \nLeftarrow$ or, equivalently, such that $\operatorname{tr} \mu=\iota_{E(S)}$. If $\mu_{S}=\iota_{S}$, then $S$ is said to be fundamental. If $\psi_{S}=\iota_{S}$, then $S$ is said to be combinatorial. Equivalently, $S$ is combinatorial if it contains no nontrivial subgroups.

LEMMA 2.1 (SEE [3]). For any inverse semigroup $S$ and any $a, b \in S$,

$$
(a, b) \in \mu_{S} \Leftrightarrow a^{-1} e a=b^{-1} e b, \quad \text { for all } e \in E(S) .
$$

An inverse semigroup $P$ is an E-unitary cover of the inverse semigroup $S$ if $P$ is $E$-unitary and there is an idempotent separating homomorphism of $P$ onto $S$; if $P / \sigma \cong G$ then $P$ is an E-unitary cover of $S$ over $G$.

A variety $\mathcal{V}$ of inverse semigroups has $E$-unitary covers if, for every $S \in \mathcal{V}$, there is an $E$-unitary cover of $S$ in $\mathcal{V}$ and $\mathcal{V}$ has E-unitary covers over a variety of groups $\mathcal{U}$ if for every $S$ in $\mathcal{V}$ there is an $E$-unitary cover of $S$ over some group in $\mathcal{U}$.

THEOREM 2.2 (SEE [7]). The following conditions on a variety $\mathcal{V}$ of inverse semigroups are equivalent.

(i) $\mathcal{V}$ has E-unitary covers.

(ii) The free objects in $\mathcal{V}$ are E-unitary.

(iii) $\mathcal{V}$ is generated by its E-unitary members.

An inverse semigroup is completely semisimple if and only if each of its principal factors is a Brandt semigroup or, equivalently, if and only if it does not contain the bicyclic semigroup $c$ as a subsemigroup (see Theorem 2.54 of $[\mathbf{1}]$ ).

THEOREM 2.3 (SEE [9]). For a variety of inverse semigroups $\mathcal{V}$, the following statements are equivalent:

(1) $C \notin \mathcal{V}$;

(2) Every member of $\mathcal{V}$ is completely semisimple;

(3) For some positive integer $n, x^{n} x^{-n}=x^{-n} x^{n}$ is a law in $\mathcal{V}$.

3. Inherited properties. In this section we begin to address the general question of when it is possible to obtain information about the structure of the relatively free objects in a variety $\mathcal{V}$ of inverse semigroups. Assuming that a detailed construction of $F V_{X}$ is not available, it is natural to ask if $F \mathcal{V}_{X}$ falls into any wellknown classes of inverse semigroups, such as $E$-unitary, completely semisimple, fundamental or combinatorial.

We identify certain properties which, if possessed by an inverse semigroup $S$, will be inherited by the relatively free objects of $\mathcal{V}(S)$.

THEOREM 3.1. Let $S$ be an inverse semigroup and $\mathcal{V}=\mathcal{V}(S)$. If $S$ is combinatorial (respectively, completely semisimple or $E$-unitary) then $F=F V_{X}$ is also combinatorial (respectively, completely semisimple or E-unitary). If $S$ is fundamental and $|X| \geq \aleph_{0}$, then so is $F \mathcal{V}_{X}$. 
ProOF. Let $\rho$ be the fully invariant congruence on $F I_{X}$ corresponding to $\mathcal{V}(S)$. Then $\rho=\bigcap\left\{\theta \circ \theta^{-1} \mid \theta: F I_{X} \rightarrow S\right.$ is a homomorphism $\}$ and $F \cong F I_{X} / \rho$.

Let $a, b \in F I_{X}$ be such that $a \rho \neq b \rho$. Then there exists a homomorphism $\theta: F I_{X} \rightarrow S$ such that $a \theta \neq b \theta$.

If $S$ is combinatorial, then it follows that $(a \theta, b \theta) \notin \mathcal{H}_{S}$. Since $\rho \subseteq \theta \circ \theta^{-1}$, it follows that $(a \rho, b \rho) \notin \mathcal{H}_{F}$. Thus $F$ is combinatorial.

Now let $S$ be completely semisimple and let $a \in F I_{X}$ be such that $(a \rho)(a \rho)^{-1} \neq$ $(a \rho)^{-1}(a \rho)$. Then, for some $\theta: F I_{X} \rightarrow S,(a \theta)(a \theta)^{-1} \neq(a \theta)^{-1}(a \theta)$. Since $S$ is completely semisimple, $(a \theta)(a \theta)^{-1}$ and $(a \theta)^{-1}(a \theta)$ are incomparable (D-equivalent) idempotents. Hence $(a \rho)(a \rho)^{-1}$ and $(a \rho)^{-1}(a \rho)$ are also incomparable. Thus $F$ cannot contain a copy of the bicyclic semigroup and so $F$ is completely semisimple.

That $F$ will be $E$-unitary if $S$ is $E$-unitary was established in [4 and $\mathbf{7}]$.

Finally suppose that $S$ is fundamental and that $|X|=\aleph_{0}$. Again let $a, b \in F I_{X}$ be such that $a \rho \neq b \rho$ so that there exists a homomorphism $\theta: F I_{X} \rightarrow S$ with $a \theta \neq b \theta$. Since $S$ is fundamental, there exists an idempotent $e \in S$ such that

$$
(a \theta)^{-1} e(a \theta) \neq(b \theta)^{-1} e(b \theta) .
$$

Let $c(a) \cup c(b)=\left\{x_{1}, \ldots, x_{n}\right\}$ and $z \in X \backslash\left\{x_{1}, \ldots, x_{n}\right\}$. Define a homomorphism $\varphi: F I_{X} \rightarrow S$ by its action on $X$ as follows:

$$
x \varphi= \begin{cases}e & \text { if } x=z \\ x \theta & \text { otherwise }\end{cases}
$$

Then

$$
\begin{aligned}
\left(a^{-1} z z^{-1} a\right) \varphi & =(a \varphi)^{-1}(z \varphi)(z \varphi)^{-1}(a \varphi)=(a \theta)^{-1} e(a \theta) \\
& \neq(b \theta)^{-1} e(b \theta)=(b \varphi)^{-1}(z \varphi)(z \varphi)^{-1}(b \varphi) \\
& =\left(b^{-1} z z^{-1} b\right) \varphi
\end{aligned}
$$

so that $\left(a^{-1} z z^{-1} a, b^{-1} z z^{-1} b\right) \notin \rho$. Therefore,

$$
(a \rho)^{-1}\left(z z^{-1}\right) \rho(a \rho) \neq(b \rho)^{-1}\left(z z^{-1}\right) \rho(b \rho)
$$

and

$$
(a \rho, b \rho) \notin \mu_{F} .
$$

Thus $F$ is fundamental.

It was shown in $[\mathbf{1 0}]$ that the free inverse semigroup on two generators contains the free inverse semigroup on a countably infinite set of generators so that, for $n \geq 2, \mathcal{V}\left(F_{n}(I)\right)=\mathcal{V}\left(F_{\aleph_{0}}(I)\right)=I$ and $F I_{X}$ is well known. However, $\mathcal{V}\left(F_{1}(I)\right)=$ $C \neq I$ (see [6, Proposition XII.4.11]), and so it is natural to ask if $F C_{X}$ has any of the properties referred to in Proposition 3.1.

COROLlaRY 3.2. For all $X \neq \emptyset, F C_{X}$ is combinatorial, completely semisimple and E-unitary.

Proof. Since $F_{1} I$ is combinatorial, completely semisimple and $E$-unitary, so also is $F C_{X}$, by Proposition 3.1.

THEOREM 3.3. Let $\mathcal{V}$ and $\mathcal{W}$ be varieties of inverse semigroups with $\mathcal{V} \subseteq \mathcal{W}$ and $\operatorname{ker} \rho \mathcal{v}=\operatorname{ker} \rho w$. If $F \mathcal{V}_{X}$ is combinatorial, fundamental or $E$-unitary, then so 
also is $F W_{X}$. If $F \mathcal{V}_{X}$ is completely semisimple and combinatorial, then $F W_{X}$ is completely semisimple.

Proof. Let $F=F \mathcal{V}_{X}$ and $F^{\prime}=F \mathcal{W}_{X}$. Since $\mathcal{V} \subseteq \mathcal{W}$, it follows that $\rho \mathcal{W} \subseteq \rho \mathcal{v}$. Since $\rho_{\mathcal{V}}$ and $\rho_{\mathcal{W}}$ have the same kernel, the congruence $\rho=\rho_{\mathcal{V}} / \rho_{\mathcal{W}}$ induced on $F^{\prime}$ by $\rho \mathcal{v}$ is necessarily idempotent pure.

Suppose that $F$ is $E$-unitary, that is, the minimum group congruence $\sigma$ on $F$ is idempotent pure. Then

$$
\tau=\left\{(a, b) \in F^{\prime} \times F^{\prime}:(a \rho, b \rho) \in \sigma\right\}
$$

is an idempotent pure congruence on $F^{\prime}$ such that $F^{\prime} / \tau \cong F / \sigma$ is a group. Hence $\tau$ is the minimum group congruence on $F^{\prime}$ and therefore $F^{\prime}$ is $E$-unitary.

Now let $F$ be combinatorial. Let $a \in F^{\prime} \backslash E\left(F^{\prime}\right)$. Since $\rho$ is idempotent pure, $a \rho \notin E(F)$. Since $F$ is combinatorial $\left(a \rho,(a \rho)(a \rho)^{-1}\right) \notin \not_{F}$. Hence $\left(a, a a^{-1}\right) \notin \nvdash_{F^{\prime}}$ and $F^{\prime}$ is combinatorial.

Now suppose that $F$ is fundamental and let $a \in F^{\prime} \backslash E\left(F^{\prime}\right)$. Then again $a \rho \in$ $F \backslash E(F)$ and so, by Lemma 2.1 , there exists an idempotent $y \in E(F)$ such that

$$
y \leq(a \rho)(a \rho)^{-1} \text { and }(a \rho)^{-1} y(a \rho) \neq y .
$$

Let $e \in E\left(F^{\prime}\right)$ be such that $y=e \rho$. Then, from (4) we have $a^{-1} e a \neq e$. By Lemma $2.1,\left(a, a a^{-1}\right) \notin \mu\left(F^{\prime}\right)$. Hence $\operatorname{ker} \mu\left(F^{\prime}\right)=E\left(F^{\prime}\right)$. Since $\operatorname{tr} \mu\left(F^{\prime}\right)$ is the identity relation on $E\left(F^{\prime}\right)$, it follows that $\mu\left(F^{\prime}\right)$ is the identity and $F^{\prime}$ is fundamental.

Now suppose that $F$ is combinatorial and completely semisimple. Let $a \in F I_{X}$ be such that $a^{-1} a \rho \mathcal{W} \leq a a^{-1} \rho \mathcal{W}$. Then $a^{-1} a \rho \mathcal{v} \leq a a^{-1} \rho \mathcal{v}$. Since $F$ is completely semisimple, $a^{-1} a \rho v=a a^{-1} \rho \mathcal{v}$. Hence $\left(a \rho v, a a^{-1} \rho \nu\right) \in \mathcal{H}$. But $F$ is combinatorial. Therefore $a \rho v=a a^{-1} \rho v$. Since $\rho v / \rho w$ is idempotent pure, it follows that $a \rho \mathcal{w}=$ $a a^{-1} \rho \mathcal{W}$. Hence there is no element $a \rho_{\mathcal{W}}$ such that $\left(a \rho_{\mathcal{W}}\right)^{-1}\left(a \rho_{\mathcal{W}}\right) \lesseqgtr\left(a \rho_{\mathcal{W}}\right)\left(a \rho_{\mathcal{W}}\right)^{-1}$ so that $F^{\prime}$ cannot contain a copy of $C$ and is, therefore, completely semisimple.

In regard to the last statement of Theorem 3.3, the fact that $F V_{X}$ is completely semisimple does not, by itself, imply that $F \mathcal{W}_{X}$ is completely semisimple. We will see this in Remark 4.3.

Notation 3.4. For any fully invariant subgroup $N$ of $F \mathfrak{G}_{X}$, let $\sigma_{N}$ denote the congruence on $F I_{X}$ such that $F I_{X} / \sigma_{N} \cong F G_{X} / N$ and let $\rho_{N}=\sigma_{N}^{\min }$, the minimum congruence on $F I_{X}$ with the same kernel as $\sigma_{N}$.

LEMMA 3.5. (i) $\rho_{N}$ is a fully invariant congruence on $F I_{X}$.

(ii) $\rho_{N}$ is the smallest congruence $\rho$ on $F I_{X}$ such that $F I_{X} / \rho$ is E-unitary over $F \mathcal{G}_{X} / N$

(iii) $\rho_{N}$ is the congruence on $F I_{X}$ generated by $\{((A, m),(A, n)): m, n \in N\}$.

PROOF. Part (i) follows from Theorem 3.6 of [5].

By the definition of $\rho_{N}, \operatorname{ker} \rho_{N}=\operatorname{ker} \sigma_{N}$ so that $\sigma_{N} / \rho_{N}$ is an idempotent pure congruence. Since $\left(F I_{X} / \rho_{N}\right) /\left(\sigma_{N} / \rho_{N}\right) \cong F I_{X} / \sigma_{N} \cong F G_{X} / N$ it follows that $F I_{X} / \rho_{N}$ is $E$-unitary over $F \mathcal{G}_{X} / N$. The minimality of $\rho_{N}$ follows from the fact that $\rho_{N}$ is the smallest congruence with the same kernel as $\sigma_{N}$. Hence (ii) holds.

Let $\tau$ be the congruence generated by the set given in the statement of (iii). Since $\operatorname{ker} \rho_{N}=\{(A, m): m \in N\}$ it follows that

$$
(A, m) \rho_{N}(A, 1) \rho_{N}(A, n) \text { for all } m, n \in N,
$$


so that $\tau \subseteq \rho_{N}$. On the other hand, it is clear that $\operatorname{ker} \rho_{N} \subseteq \operatorname{ker} \tau$. Hence $\operatorname{ker} \rho_{N}=$ $\operatorname{ker} \tau$. But $\tau \subseteq \rho_{N}$ while $\rho_{N}$ is the minimum congruence with its kernel. Therefore $\rho_{N}=\tau$.

Notation 3.6. For any variety $\mathcal{U}$ of groups, corresponding to the fully invariant subgroup $N$ of $F g_{X}$, let

$$
\mathcal{U}^{\max }=\mathcal{V}\left(F I_{X} / \rho_{N}\right)
$$

THEOREM 3.7. Let $\mathcal{U}$ and $N$ be as in Notation 3.6 and let $\mathcal{W}=\mathcal{U}^{\max }$.

(i) $W$ is the largest variety such that $\operatorname{ker} \rho_{\mathcal{W}}=\operatorname{ker} \sigma_{N}$.

(ii) $\mathcal{W}$ is the variety for which $\rho_{\mathcal{W}}$ is the smallest fully invariant congruence with $\operatorname{ker} \rho w=\operatorname{ker} \sigma_{N}$.

(iii) $\mathcal{W}$ is the largest variety with E-unitary covers over $\mathcal{U}$.

PROOF. By the definition of $\mathcal{W}=\mathcal{U}^{\max }$, it is clear that $\rho_{\mathcal{W}}=\rho_{N}$. Then (i) follows from the definition of $\rho_{N}$. Part (ii) is equivalent to (i).

By Lemma 3.5(ii), $F \mathcal{W}_{X}$ is $E$-unitary, for all nonempty sets $X$. By Theorem 2.2, $\mathcal{W}$ has $E$-unitary covers which will be over the variety $\mathcal{G} \cap \mathcal{W}=\mathcal{U}$. On the other hand, if $\mathcal{V}$ has $E$-unitary covers over $U$, then $F V_{X}$ is $E$-unitary over $U$. Hence $\operatorname{ker} \rho \mathcal{V}=\operatorname{ker} \rho \mathcal{u}=\operatorname{ker} \sigma_{N}$. Since $\rho_{\mathcal{W}}$ is the minimum congruence with $\operatorname{ker} \rho_{\mathcal{W}}=$ $\operatorname{ker} \sigma_{N}$, it follows that $\rho_{\mathcal{W}} \subseteq \rho_{\mathcal{V}}$ and so $\mathcal{V} \subseteq \mathcal{W}$.

Recalling that $C$ is the variety generated by the bicyclic semigroup, it follows from Corollary 3.2 and [6, Theorem XII.6.9] that

$$
A G \subseteq C \subseteq A G^{\max } .
$$

COROLlaRY 3.8. For any variety $\mathcal{V}$ such that $C \subseteq \mathcal{V} \subseteq A G^{\max }, F \mathcal{V}_{X}$ is combinatorial, completely semisimple and E-unitary over $A G$.

ProOF Since $A G \subseteq C \subseteq \mathcal{V} \subseteq A G^{\text {max }}$, it follows that ker $\rho_{\mathcal{V}}=\operatorname{ker} \rho_{C}$. The claim now follows from Corollary 3.2 and Theorem 3.3.

We will see in $\S 6$, that the interval $\left[C, A G^{\max }\right]$ contains at least $\aleph_{0}$ distinct members.

Proposition 3.9. Let $\mathcal{U}, \mathcal{V}, \mathcal{W}$ be varieties such that

(i) $U \subseteq \mathcal{V}$,

(ii) $\operatorname{tr} \rho u \subseteq \operatorname{tr} \rho w$,

Then

(iii) $\operatorname{ker} \rho v=\operatorname{ker}(\rho \nu \vee \rho w)$.

$$
\mathcal{V} \cap(\mathcal{U} \vee \mathcal{W})=\mathcal{U} \vee(\mathcal{V} \cap \mathcal{W})
$$

PROOF. Let $\beta=\rho \mathcal{v} \vee\left(\rho_{\mathcal{U}} \cap \rho_{\mathcal{W}}\right), \gamma=\rho_{\mathcal{u}} \cap\left(\rho_{\mathcal{V}} \vee \rho_{\mathcal{W}}\right)$. We wish to show that $\beta=\gamma$.

By (i), $\rho \nu \subseteq \rho u$, from which it follows easily that $\beta \subseteq \gamma$.

Define the relation $\theta$ on the lattice $\Lambda(S)$ of congruences on $S$ by

$$
(\rho, \sigma) \in \theta \Leftrightarrow \operatorname{tr} \rho=\operatorname{tr} \sigma .
$$

Then, by [6, Chapter III], $\theta$ is a congruence relation on $\Lambda(S)$. Part (ii) of the hypothesis then implies that $\rho u \theta \leq \rho \mathcal{w}$. Therefore,

$$
\begin{aligned}
\beta \theta & =\left[\rho_{\nu} \vee\left(\rho_{u} \cap \rho_{\mathcal{W}}\right)\right] \theta=\rho_{\mathcal{v}} \theta \vee\left(\rho_{\mathcal{u}} \theta \wedge \rho_{\mathcal{w}} \theta\right) \\
& =\rho_{\nu} \theta \vee \rho_{\mathcal{u}} \theta=\left(\rho_{\nu} \vee \rho_{\mathcal{u}}\right) \theta=\rho_{\mathcal{u}} \theta .
\end{aligned}
$$


On the other hand, by (ii)

so that

$$
\rho_{\mathcal{U}} \theta \leq \rho_{\mathcal{W}} \theta \leq\left(\rho_{\mathcal{V}} \vee \rho_{\mathcal{W}}\right) \theta
$$

$$
\gamma \theta=\rho_{u} \theta \wedge\left(\rho v \vee \rho_{w}\right) \theta=\rho_{u} \theta .
$$

Thus $\beta \theta=\gamma \theta$ or $\operatorname{tr} \beta=\operatorname{tr} \gamma$. Also

$$
\begin{aligned}
\operatorname{ker} \gamma & =\operatorname{ker}\left(\rho_{\mathcal{U}} \cap\left(\rho_{\mathcal{V} \vee} \vee \mathcal{W}\right)\right) \\
& =\operatorname{ker} \rho_{\mathcal{U}} \cap \operatorname{ker}\left(\rho \mathcal{v} \vee \rho_{\mathcal{W}}\right) \\
& =\operatorname{ker} \rho \mathcal{u} \cap \operatorname{ker} \rho \mathcal{v} \quad \text { by (iii) } \\
& =\operatorname{ker} \rho \mathcal{v} \quad \text { since } \rho \mathcal{v} \subseteq \rho_{\mathcal{U}} .
\end{aligned}
$$

On the other hand, it is clear that

$$
\operatorname{ker} \rho \nu \subseteq \operatorname{ker} \beta .
$$

Thus ker $\gamma \subseteq \operatorname{ker} \beta$, so that $\gamma \subseteq \beta$. Hence $\beta=\gamma$.

Notation 3.10. For any $u \in F I_{X}$, let $l(u)$ denote the length of $\bar{u}$ in reduced form. Let

$$
I_{n}=\left\{u \in F I_{1}: l(u) \geq n\right\},
$$

and let

$$
M_{n}=F I_{1} / I_{n}, \quad n=2,3, \ldots
$$

Problem XII.6.12(iii) of $[\mathbf{6}]$ poses the question: Is

$$
\mathcal{C} \cap\left(\mathcal{V}\left(M_{n}\right) \vee \mathcal{G}\right)=\mathcal{V}\left(M_{n}\right) \vee(\mathcal{C} \cap \mathcal{G}) ?
$$

We can use Proposition 3.9 to answer this affirmatively.

Since conditions (ii) and (iii) of Proposition 3.9 are satisfied automatically whenever $\mathcal{W}=\mathcal{G}$ and $F \mathcal{V}_{X}$ is $E$-unitary, we have the following

COROllary 3.11. Let $\mathcal{U}, \mathcal{V}$ be varieties such that $\mathcal{U} \subseteq \mathcal{V}$ and $F \mathcal{V}_{X}$ is Eunitary. Then

$$
\mathcal{V} \cap(\mathcal{U} \vee \mathcal{G})=\mathcal{U} \vee(\mathcal{V} \cap \mathcal{G})
$$

In particular, if $\mathcal{U}=\mathcal{V}\left(M_{n}\right)$ and $\mathcal{V}=\mathcal{C}$ then

$$
\mathcal{C} \cap\left(\mathcal{V}\left(M_{n}\right) \vee \mathcal{G}\right)=\mathcal{V}\left(M_{n}\right) \vee(C \cap G)=\mathcal{V}\left(M_{n}\right) \vee A G .
$$

4. A noncompletely semisimple example. Every relatively free inverse semigroup that has been studied in the literature in any depth to date is completely semisimple and it has long been conjectured that all relatively free inverse semigroups might be completely semisimple. This section is devoted to a remarkable example, due to P. G. Trotter, of a relatively free inverse semigroup which is not completely semisimple.

THEOREM 4.1. Let $x, y \in X, x \neq y$, and let

$$
a=x y x y(x y x y)^{-1} x y x^{-1} y^{-1} \in F I_{X} .
$$

Let $\mathcal{V}$ be the variety defined by the identity

$$
a a^{-1}=a^{2} a^{-2} .
$$

Then $F \mathcal{V}_{X}$ is not completely semisimple. 
PROOF. Let $g=a \rho \nu$. Then clearly $g g^{-1}=g^{2} g^{-2}$. Moreover, by (5),

$$
\begin{aligned}
\left(g^{-1} g\right)\left(g g^{-1}\right) & =\left(g^{-1} g\right)\left(g g^{-1}\right)\left(g^{-1} g\right)=g^{-1} g^{2} g^{-2} g \\
& =g^{-1} g g^{-1} g=g^{-1} g .
\end{aligned}
$$

Thus $g^{-1} g \leq g g^{-1}$. Our objective, therefore, is to show that

$$
g^{-1} g \neq g g^{-1}
$$

since it will then follow from [1, Lemma 1.31], that the inverse subsemigroup of $F I_{X} / \rho v=F V_{X}$ generated by $g$ is isomorphic to the bicyclic semigroup so that $F \mathcal{V}_{X}$ is not completely semisimple.

The relation

$$
\delta=\left\{\left(\left(a a^{-1}\right) \varphi,\left(a^{2} a^{-2}\right) \varphi\right): \varphi \text { is an endomorphism of } F I_{X}\right\}
$$

is clearly a fully invariant relation on $F I_{X}$. By Lemma 2.2 of $[\mathbf{5}]$, the congurence $\rho$ generated by $\delta$ is a fully invariant congruence. Now $\rho v$ is the smallest fully invariant congruence on $F I_{X}$ containing $\left(a a^{-1}, a^{2} a^{-2}\right)$ so that $\rho \mathcal{V} \subseteq \rho$. On the other hand, $\delta \subseteq \rho \nu$ so that $\rho \subseteq \rho \nu$. Hence $\rho \mathcal{v}=\rho$ is the congruence generated by $\delta$.

It follows easily that $\left(a a^{-1}, a^{n} a^{-n}\right) \in \rho \mathcal{\nu}$, for all $n \geq 1$. Now

$$
A(a)=A\left(a a^{-1}\right)=\left\{1, x, x y, x y x, x y x y, x y x^{-1}, x y x^{-1} y^{-1}\right\}
$$

and, writing $h=\bar{a}$, we have

$$
h=\bar{a}=x y x^{-1} y^{-1} .
$$

In Scheiblich form,

$$
a=(A(a), h) \quad \text { and } \quad a a^{-1}=(A(a), 1) .
$$

By repeated applications of (3), we have, for $m \geq 1$,

$$
\begin{aligned}
A\left(a^{m}\right) & =A\left(a^{m} a^{-m}\right)=\bigcup_{i=0}^{m-1} h^{i} A(a) \\
& =\left\{h^{i}, h^{i} x, h^{i} x y, h^{i} x y x, h^{i} x y x y, h^{i} x y x^{-1}, h^{i+1}: i=0, \ldots, m-1\right\} .
\end{aligned}
$$

The main work of the proof lies in the following

Lemma 4.2. Let $b \in F I_{X}$ and $\left(a a^{-1}, b b^{-1}\right) \in \rho \mathcal{V}$. Then $A(b) \subseteq A\left(a^{m}\right)$, for some $m \geq 1$.

PROOF. Since $\rho v$ is the congruence generated by $\delta$, there exist elements $p_{i}, a_{i}, b_{i}$ $\in F I_{X}, i=0, \ldots, n$, with $p_{0}=a_{0}=b_{0}=a a^{-1}$ such that

$$
\begin{aligned}
a a^{-1} & =p_{0} b_{0} p_{0}^{-1}, \\
p_{j} a_{j} p_{j}^{-1} & =p_{j+1} b_{j+1} p_{j+1}^{-1}, \quad j=0, \ldots, n-1, \\
p_{n} a_{n} p_{n}^{-1} & =b b^{-1},
\end{aligned}
$$

where $\left(a_{i}, b_{i}\right) \in \delta \cup \delta^{-1} \cup \iota$ for $0 \leq i \leq n$. Note that, by the definition of $\delta$ we may assume that each $a_{i}$ and each $b_{i}$ is an idempotent. 
The proof is by induction on $n$. If $n=0$, then

$$
a a^{-1}=p_{0} a_{0} p_{0}^{-1}=p_{0} a_{0} p_{0}^{-1}=b b^{-1}
$$

and the claim holds. So suppose that the claim holds for all sequences of the form (8) of shorter length and consider the sequence (8).

Since the subsequence of $(8)$ up to

$$
p_{n-1} a_{n-1} p_{n-1}^{-1}=p_{n} b_{n} p_{n}^{-1}
$$

is of shorter length, we may assume that

$$
A\left(p_{n} b_{n} p_{n}^{-1}\right) \subseteq A\left(a^{m}\right) \text { for some } m \geq 1 .
$$

As observed above we can assume that each $b_{n}$ is an idempotent so that $\bar{b}_{n}=1$. Then, by (2) and (3) and writing $P=A\left(p_{n}\right)$,

$$
\begin{aligned}
A\left(p_{n} b_{n} p_{n}^{-1}\right) & =A\left(p_{n}\right) \cup \bar{p}_{n} A\left(b_{n}\right) \cup \bar{p}_{n} \bar{b}_{n} A\left(p_{n}^{-1}\right) \\
& =P \cup \bar{p}_{n} A\left(b_{n}\right) \cup \bar{p}_{n} \bar{p}_{n}^{-1} A\left(p_{n}\right) \\
& =P \cup \bar{p}_{n} A\left(b_{n}\right) .
\end{aligned}
$$

Therefore, by (9), we have

$$
P \cup \bar{p}_{n} A\left(b_{n}\right) \subseteq A\left(a^{m}\right) .
$$

Now $\left(a_{n}, b_{n}\right) \in \delta \cup \delta^{-1} \cup \iota$. If $a_{n}=b_{n}$, then the conclusion holds easily. So suppose that $\left(a_{n}, b_{n}\right) \in \delta \cup \delta^{-1}$. Then, for some endomorphism $\varphi$ of $F I_{X}$,

$$
a_{n}, b_{n} \in\left\{a a^{-1} \varphi, a^{2} a^{-2} \varphi\right\} .
$$

Let $x \varphi=d$ and $y \varphi=e$ and $t=a \varphi=\operatorname{dede}(\text { dede })^{-1} \operatorname{ded}^{-1} e^{-1}$. Then

$$
a_{n}, b_{n} \in\left\{t t^{-1}, t^{2} t^{-2}\right\}
$$

so that there are two cases to consider.

Case (i). $b_{n}=t^{2} t^{-2}, a_{n}=t t^{-1}$. Then, by (3),

$$
\begin{aligned}
A(b) & =A\left(p_{n} a_{n} p_{n}^{-1}\right)=A\left(p_{n} t t^{-1} p_{n}^{-1}\right) \\
& =A\left(p_{n} t\right) \subseteq A\left(p_{n} t^{2}\right) \\
& =A\left(p_{n} t^{2} t^{-2} p_{n}^{-1}\right)=A\left(p_{n} b_{n} p_{n}^{-1}\right) \subseteq A\left(a^{m}\right)
\end{aligned}
$$

and the result holds.

Case (ii). $b_{n}=t t^{-1}, a_{n}=t^{2} t^{-2}$. Let $p=p_{n}$. Then, by (10)

$$
P \cup \bar{p} A(t)=P \cup \bar{p} A\left(b_{n}\right) \subseteq A\left(a^{m}\right),
$$

for some $m \geq 1$, while

$$
A(b)=A\left(p t^{2} t^{-2} p^{-1}\right)=P \cup \bar{p} A(t) \cup \bar{p} \bar{t} A(t) .
$$

From this and (11), we see that it remains to show that

$$
\bar{p} \bar{t} A(t) \subseteq A\left(a^{m}\right) \quad \text { for some } m .
$$

This will follow from (11), if we can show that, for all choices of $\varphi$,

$$
\text { either }(\alpha) \bar{t}=1 \text { or }(\beta) \bar{p} \text { and } \bar{t} \text { are both powers of } h=\bar{a} \text {. }
$$


Note that, in $F G_{X}$,

$$
\bar{t}=\bar{d} \bar{e} \bar{d}^{-1} \bar{e}^{-1}
$$

Let

$$
u=\bar{p} \bar{d} \bar{e}, \quad v=\bar{p} \bar{d} \bar{e} \bar{d}, \quad w=\bar{p} \bar{d} \bar{e} \bar{d} \bar{e} .
$$

Now it follows easily from the definition of $t$ that

$$
1, \bar{d}, \bar{d} \bar{e}, \bar{d} \bar{e} \bar{d}, \bar{d} \bar{e} \bar{d} \bar{e}, \bar{d} e \bar{d}^{-1}, \bar{d} \bar{e} \bar{d}^{-1} \bar{e}^{-1} \in A(t)
$$

so that, by (11),

$$
\bar{p}, \bar{p} \bar{d}, u, v, w, \bar{p} \bar{d} \bar{d} \bar{d}^{-1} \bar{e}^{-1}, u \bar{p}^{-1} u, u v^{-1} u, u w^{-1} u \in A\left(a^{m}\right) .
$$

Also, for future reference we note that

$$
\bar{d}=u^{-1} v \quad \text { and } \quad \bar{e}=v^{-1} w .
$$

Now proceed by considering all the possible combinations of values for $p, u, v, w$ from (7), since these elements all lie in $A\left(a^{m}\right)$, for some $m \geq 1$, by (13). This requires an exhaustive case-by-case argument. We only include here a few sample cases, including the hardest. (The others may be found in the appendix which is obtainable from the authors.) We focus on the possible values of $u$ in $A\left(a^{m}\right)$. The key to the argument is the fact that for $z=\bar{p}, v, w$ we also have

$$
z \text { and } u z^{-1} u \in A\left(a^{k}\right), \quad \text { for some } k>0 .
$$

We begin with an easy illustrative case.

(i) $u=\bar{a}^{i}=h^{i}$, for some $i \geq 0$.

As $z$ runs through the possible values in

$$
A\left(a^{k}\right)=\left\{h^{j}, h^{j} x, h^{j} x y, h^{j} x y x, h^{j} x y x y, h^{j} x y x^{-1}, h^{j+1} ; j=0, \ldots, k-1\right\}
$$

then $u z^{-1} u$ runs through the values of the form

$$
\begin{aligned}
& h^{2 i-j}, h^{i} x^{-1} h^{i-j}, h^{i}(x y)^{-1} h^{i-j}, h^{i}(x y x)^{-1} h^{i-j}, \\
& h^{i}(x y x y)^{-1} h^{i-j}, h^{i}\left(x y x^{-1}\right)^{-1} h^{i-j} .
\end{aligned}
$$

Of these, only $h^{2 i-j}(j \leq 2 i)$ lies in $A\left(a^{k}\right)$, for some $k$. Therefore, $z=h^{j}$, for some $j \geq 0$, so that $u, v$ and $w$ are all of the form $h^{j}$. Hence $\bar{d}$ and $\bar{e}$ are also of this form, by (14), and therefore $\bar{t}=1$.

The cases where $u=h^{i} x, h^{i} x y x, h^{i} x y x y$ or $h^{i} x y x^{-1}$ are not much more complicated than Case (i). However, the case $u=h^{i} x y$ has many subcases. We consider a few:

(ii) $u=h^{i} x y, i \geq 0$. In this case, each possible value of $z \in A\left(a^{k}\right)$ requires a separate consideration.

(a) $z=h^{j}, j \geq 0$. Then $u z^{-1} u=h^{i} x y h^{i-j} x y$. If $i>j$, then this is in reduced form and cannot lie in $A\left(a^{k}\right)$, for any $k \geq 0$. If $i<j$ then

$$
\begin{aligned}
u z^{-1} u & =h^{i} x y h^{i-j+1}\left(y x y^{-1} x^{-1}\right) x y \\
& =h^{i} x y h^{i-j+1} y x \notin A\left(a^{k}\right) \text { for any } k \geq 0 .
\end{aligned}
$$

If $i=j$, then $u z^{-1} u=h^{i} x y x y \in A\left(a^{m}\right)$. Thus $z=h^{i}$ is a possibility.

(b) $z=h^{j} x, j \geq 0$. Then $u z^{-1} u=h^{i} x y x^{-1} h^{i-j} x y$. If $i=j$, then this becomes $u z^{-1} u=h^{i} x y y \notin A\left(a^{k}\right), \quad$ for any $k$. 
If $i<j$, then we obtain

$$
\begin{aligned}
u z^{-1} u & =h^{i} x y x^{-1} h^{i-j+1} y x y^{-1} x^{-1} x y \\
& =h^{i} x y x^{-1} h^{i-j+1} y x
\end{aligned}
$$

in reduced form, which does not lie in $A\left(a^{k}\right)$ for any $k$. If $i>j$, then we obtain

$$
\begin{aligned}
u z^{-1} u & =h^{i} x y x^{-1} x y x^{-1} y^{-1} h^{i-j-1} x y \\
& =h^{i} x y y x^{-1} y^{-1} h^{i-j-1} x y \notin A\left(a^{k}\right), \text { for any } k .
\end{aligned}
$$

Thus $z=h^{i} x$ is not possible.

Investigating in a similar way the cases $z=h^{j} x y, h^{j} x y x, h^{j} x y x y$ and $h^{j} x y x^{-1}$ we find that the possible values for $z$ (that is, $\bar{p}, v$ or $w$ ) are

$$
h^{i}, h^{j} x y(j \leq 2 i), h^{i} x y x, h^{i} x y x y, h^{i} x y x^{-1} .
$$

We must now consider each of these possibilities in turn. We focus on the value of $v$. Of course, we still have $u=h^{i} x y, i \geq 0$.

(1) $v=h^{i}$. Then from (14)

$$
\bar{d}=u^{-1} v=(x y)^{-1} .
$$

Now $\bar{p}$ is one of the elements listed in (15) so that $\bar{p} \bar{d}$ is of one of the forms

$$
h^{i}(x y)^{-1}, h^{j}, h^{i} x y x y^{-1} x^{-1}, h^{i} x y, h^{i} x y x^{-1} y^{-1} x^{-1} .
$$

By (13), $\bar{p} \bar{d} \in A\left(a^{m}\right)$. Hence we must have

$$
\bar{p} \bar{d}=h^{j} \quad \text { or } \quad h^{i} x y,
$$

that is, either $\bar{p}=h^{j} x y$ or $\bar{p}=h^{i} x y x y$.

If $\bar{p}=h^{i} x y x y$, then

$$
h^{i} x y=u=\bar{p} \bar{d} \bar{e}=h^{i} x y x y(x y)^{-1} \bar{e}=h^{i} x y \bar{e}
$$

so that $\bar{e}=1$ and $\bar{t}=1$, which is satisfactory.

So suppose that $\bar{p}=h^{j} x y$. Then

$$
h^{i} x y=u=\bar{p} \bar{d} \bar{e}=h^{j} x y(x y)^{-1} \bar{e}
$$

so that $\bar{e}=h^{i-j} x y$. But then

$$
\bar{p} \bar{d} \bar{e} \bar{d}^{-1} \bar{e}^{-1}=h^{j} x y(x y)^{-1} h^{i-j} x y(x y)(x y)^{-1} h^{j-i}=h^{i} x y h^{j-i}
$$

which, by (13), must lie in $A\left(a^{m}\right)$. This can only happen if $j=i$. Then $\bar{e}=x y, \bar{d}=$ $(x y)^{-1}$ and again $\bar{t}=1$.

(2) $v=h^{j} x y$. Then $\bar{d}=u^{-1} v=(x y)^{-1} h^{j-i} x y$.

If $j=i$, then $\bar{d}=1$ so that $\bar{t}=1$. So suppose first that $j>i$. Then

$$
\begin{aligned}
\bar{d} & =(x y)^{-1} h^{j-i} x y=(x y)^{-1} x y x^{-1} y^{-1} h^{j-i-1} x y \\
& =x^{-1} y^{-1} h^{j-i-1} x y .
\end{aligned}
$$

From (15), $\bar{p}$ must be of one of the form

$$
h^{i}, h^{k} x y, h^{i} x y x, h^{i} x y x y, h^{i} x y x^{-1} .
$$


Then $\bar{p} \bar{d} \in A\left(a^{m}\right)$ only if $\bar{p}=h^{k} x y$. In this case

$$
\begin{aligned}
e & =\bar{d}^{-1} \bar{p}^{-1} u=(x y)^{-1} h^{i-j} x y(x y)^{-1} h^{-k} h^{i} x y \\
& =(x y)^{-1} h^{2 i-j-k} x y .
\end{aligned}
$$

Hence $\bar{e} \bar{d}=\bar{d} \bar{e}$ and $\bar{t}=1$.

So now suppose that $i>j$. Then

$$
\begin{aligned}
\bar{d} & =(x y)^{-1} h^{j-i} x y=(x y)^{-1} h^{j-i+1} h^{-1} x y \\
& =(x y)^{-1} h^{j-i+1} y x y^{-1} x^{-1} x y=(x y)^{-1} h^{j-i+1} y x .
\end{aligned}
$$

In order that $\bar{p} \bar{d}$ lie in $A\left(a^{m}\right)$, the initial $(x y)^{-1}$ of $\bar{d}$ must cancel and so, by (15), we must have

$$
\bar{p}=h^{k} x y \quad \text { or } \quad \bar{p}=h^{i} x y x y .
$$

But, if $\bar{p}=h^{i} x y x y$, then

$$
\bar{p} \bar{d}=h^{i} x y h^{j-i+1} y x
$$

which does not lie in $A\left(a^{m}\right)$. The only remaining possibility is $\bar{p}=h^{k} x y$. Then, since $\bar{d}=(x y)^{-1} h^{j-i} x y$, we have

$$
\begin{aligned}
\bar{e} & =\bar{d}^{-1} \bar{p}^{-1} u \\
& =(x y)^{-1} h^{i-j} x y(x y)^{-1} h^{-k} h^{i} x y \\
& =(x y)^{-1} h^{2 i-j-k} x y .
\end{aligned}
$$

Thus $\bar{e}$ and $\bar{d}$ are both conjugates of powers of $h$ by $x y$ so that $\bar{e} \bar{d}=\bar{d} \bar{e}$ and $\bar{t}=1$.

The remaining cases where $v=h^{i} x y x, h^{i} x y x y, h^{i} x y x^{-1}$ are all similar. (One possibility when $v=h^{i} x y x$ leads to the outcome $p=h^{i}$ and $\bar{t}=h=\bar{a}$. In all other cases $\bar{t}=1$.)

Once all possible cases are checked (for full details see the appendix) the proof of the lemma is complete and we can return to the proof of Theorem 4.1.

Now

$$
\begin{aligned}
A\left(a^{-1}\right) & =\bar{a}^{-1} A(a)=y x y^{-1} x^{-1} A(a) \\
& =\left\{1, y, y x, y x^{2}, y x^{2} y, y x y^{-1}, y x y^{-1} x^{-1}\right\}
\end{aligned}
$$

so that $A\left(a^{-1}\right) \nsubseteq A\left(a^{m}\right)$ for any $m \geq 1$. Hence, from Lemma 4.2 , we see that

$$
\left(a^{-1} a, a a^{-1}\right) \notin \rho \mathcal{v}
$$

so that $g^{-1} g \neq g g^{-1}$ and (6) holds. This completes the proof of Theorem 4.1.

REMARK 4.3. Let $\mathcal{V}$ be the variety defined in Theorem 4.1. Since any group satisfies the identity (5), it follows that $\mathcal{G} \subseteq \mathcal{V}$. Hence

$$
\operatorname{ker} \rho_{\mathcal{G}}=E\left(F I_{X}\right)=\operatorname{ker} \rho \mathcal{v} .
$$

Also $\mathcal{G}$ is a variety of completely semisimple semigroups. Thus the hypotheses of the last claim in Theorem 3.3 are satisfied except that $F g_{X}$ is not combinatorial. We see from Theorem 4.1, that $F V_{X}$ is not completely semisimple and therefore some additional assumption such as " $F V_{X}$ is combinatorial" is required in order to be able to deduce in Theorem 3.3 that $F \mathcal{W}_{X}$ is completely semisimple. 
5. Minimal varieties with fundamental free objects. Theorems 3.1 and 3.3 enable us to deduce properties of the relatively free objects in one variety from the properties of those in a related variety. In order to take advantage of this it is important to have a good set of reference points. In this section we consider just such a family of varieties.

It is convenient at this point to introduce a special case of the construction introduced in $[\mathbf{1 1}]$.

For any nonempty set $I$, let $\mathcal{M}(I)$ dencte the combinatorial Brandt semigroup $\mathcal{M}^{0}(I,\{1\}, I ; \Delta)$. For any group $G$, let

$$
\mathcal{N}(G, G)=G \cup \mathcal{M}(G)
$$

be the ideal extension of $\mathcal{M}(G)$ by $G$ (with a zero adjoined) for which products in $G$ and $\mathcal{M}(G)$ are as given while for $g \in G,(x, 1, y) \in \mathcal{M}(G)$

$$
g(x, 1, y)=\left(x g^{-1}, 1, y\right), \quad(x, 1, y) g=(x, 1, y g), \quad g 0=0=0 g .
$$

LEMMA 5.1 (SEE $[\mathbf{1 1}])$. For any group $G, \mathcal{N}(G, G)$ is a fundamental inverse semigroup and satisfies the identity $x^{2} x^{-2}=x^{-2} x^{2}$.

Proposition 5.2. Let $G$ be a group, $S=\mathcal{N}(G, G), \mathcal{U}=\mathcal{V}(G), \mathcal{V}=\mathcal{V}(S)$ and $X$ be a nonempty set.

(i) $F \mathcal{V}_{X}$ is E-unitary and completely semisimple.

(ii) If $|X| \geq \aleph_{0}$, then $F \mathcal{V}_{X}$ is fundamental.

(iii) $\mathcal{V} \subseteq \mathcal{U}^{\max }$.

Proof. Part (ii) follows from Lemma 5.1 and Theorem 3.1. That $F \mathcal{V}_{X}$ is completely semisimple follows from Lemma 5.1 and Theorems 2.3 and 3.1. We now consider the first part of (i).

Let $G_{0}=G$ and $T=\{S \backslash\{0\}\} \cup G_{0}$. We define a multiplication on $T$. The product of any two elements in $S \backslash\{0\}$ is as before provided it is nonzero. The product of any two elements in $G_{0}$ is as before. Other products are defined as follows: for $(a, 1, b),(c, 1, d) \in S \backslash\{0\}, g \in G_{0}, u \in G$,

$$
\begin{aligned}
& (a, 1, b)(c, 1, d)=a^{-1} b c^{-1} d \in G_{0} \quad(\text { if } b \neq c), \\
& g(a, 1, b)=g a^{-1} b, \\
& (a, 1, b) g=a^{-1} b g, \\
& g u=g u \in G_{0}, \quad u g=u g \in G_{0} .
\end{aligned}
$$

Then $T$ is an inverse semigroup, in fact, an ideal extension of $G_{0}$ by $S$. Thus $S$ is isomorphic to the Rees quotient $T / G_{0}$. Moreover, the natural homomorphism $\nu: T \rightarrow T / G_{0} \cong S$ separates the idempotents of $T$.

Let $\theta: T \rightarrow G_{0}=G$ be defined by:

$$
\begin{aligned}
g \theta & =g & & (g \in G), \\
(a, 1, b) \theta & =a^{-1} b & & ((a, 1, b) \in S \backslash\{0\}), \\
h \theta & =h & & \left(h \in G_{0}\right) .
\end{aligned}
$$

Then $\theta$ is an epimorphism such that the kernel of the congruence $\theta \circ \theta^{-1}$ is $E(T)$. Since $G$ is a group it follows that $T$ is $E$-unitary. 
Now let $\varphi$ be the homomorphism of $T$ into $S \times G$ defined by

$$
x \varphi=(x \nu, x \theta) .
$$

Since $\nu$ separates the idempotents of $T$ and the kernel of $\theta \circ \theta^{-1}$ is $E(T)$, it follows that the congruence $\varphi \circ \varphi^{-1}$ has trivial trace and trivial kernel. Hence $\varphi \circ \varphi^{-1}$ is the identity congruence and $\varphi$ embeds $T$ in $S \times G$. Hence $T \in \mathcal{V}(S \times G)=\mathcal{V}$. On the other hand, since $S \cong T / G_{0}$ we have $S \in \mathcal{V}(T)$. Therefore $\mathcal{V}=\mathcal{V}(T)$. But $T$ is $E$-unitary and so, by Theorem $2.2, F \mathcal{V}_{X}$ is also $E$-unitary. This establishes the other half of (i).

Since $T$ is $E$-unitary over $G$, we have $T \in \mathcal{U}^{\max }$ and therefore, $\mathcal{V}=\mathcal{V}(T) \subseteq \mathcal{U}^{\max }$ so that (iii) holds.

COROLlaRY 5.3. Let $\mathcal{V}$ be a variety of inverse semigroups with E-unitary covers and let $\mathcal{U}=\mathcal{G} \cap \mathcal{V}$. If $\mathcal{N}\left(F U_{X}, F U_{X}\right) \in \mathcal{V}$ and $|X| \geq \aleph_{0}$, then $F \mathcal{V}_{X}$ is fundamental.

Proof. Let $\mathcal{W}=\mathcal{V}\left(\mathcal{N}\left(F U_{X}, F U_{X}\right)\right)$. By Theorem 2.2, FV V is $E$-unitary so that $\operatorname{ker} \rho \mathcal{V}=\operatorname{ker} \rho_{\mathcal{U}}$. By Proposition 5.2, $\mathcal{U} \subseteq \mathcal{W} \subseteq \mathcal{U}^{\max }$. Hence $\operatorname{ker} \rho_{\mathcal{W}}=\operatorname{ker} \rho_{\mathcal{U}}=$ ker $\rho v$. The result now follows from Theorem 3.3 .

COROLlaRY 5.4. Let $\mathcal{U}$ be a variety of groups, $\mathcal{W}=\mathcal{U}^{\max }$ and $|X| \geq \aleph_{0}$. Then $F \mathcal{W}_{X}$ is fundamental.

Proof. By Theorem 3.7, $\mathcal{W}$ has $E$-unitary covers and $\mathcal{U}=\mathcal{W} \cap \mathcal{G}$. By Proposition 5.2(iii), $\mathcal{N}\left(F U_{X}, F U_{X}\right) \in \mathcal{W}$. The result now follows from Corollary 5.3.

Notation 5.5. For any prime $p$, let $Z_{p}$ denote the additive group of integers modulo $p$ and let $S_{p}=\mathcal{N}\left(Z_{p}, Z_{p}\right)$ and $\mathcal{V}_{p}=\mathcal{V}\left(S_{p}\right)$.

We can now show that the restriction on the cardinality of $X$ in Theorem 3.1, in order to obtain the conclusion in the case when $S$ is fundamental, is indeed necessary.

LEMMA 5.6. For any prime $p \geq 2, F_{n}\left(\mathcal{V}_{p}\right)$ is not fundamental.

Proof. Let $u=\left(x_{1} \cdots x_{n}\right)^{2}, v=\left(x_{1} \cdots x_{n}\right)^{3} \in F_{n}(I)$. The homomorphism $\theta: F_{n}(I) \rightarrow S_{p}$ defined by $x_{1} \theta=1 \in Z_{p}, x_{i} \theta=0 \in Z_{p}(i \neq 1)$ is such that $u \theta \neq v \theta$. Hence, if $\rho$ is the fully invariant convergence on $F_{n}(I)$ such that $F_{n}(I) / \rho \cong F_{n}\left(\mathcal{V}_{p}\right)$, then $u \rho \neq v \rho$.

Now consider any homomorphism $\theta: F_{n}(I) \rightarrow S_{p}$ and let $T=F_{n}(I) \theta$. Suppose there exists an $i$ such that $x_{i} \theta \notin Z_{p}$. Then either $\left(x_{1} \cdots x_{n}\right) \theta$ is an idempotent in $\mathcal{M}\left(Z_{p}\right)$ in which case $u \theta=v \theta$ or $\left(x_{1} \cdots x_{n}\right) \theta$ is not an idempotent, in which case $u \theta=0=v \theta$. In both of these cases $(u \theta, v \theta) \in \mu_{T}$. Now suppose that $x_{i} \theta \in Z_{p}$, for all $i$. Then $F_{n}(I) \theta \subseteq Z_{p}$ and again $(u \theta, v \theta) \in \mu_{T}$. Hence, since $\rho=\bigcap\left\{\theta \circ \theta^{-1}: \theta\right.$ is a homomorphism of $F_{n}(I)$ into $\left.S_{p}\right\},(u \rho, v \rho) \in \mu$, in $F_{n}\left(\mathcal{V}_{p}\right)$, although $u \rho \neq v \rho$. Thus $F_{n}\left(\mathcal{V}_{p}\right)$ is not fundmental.

By Lemma 5.1, the inverse semigroups $\mathcal{N}\left(Z_{p}, Z_{p}\right), p$ a prime, and $\mathcal{N}(Z, Z)$ are fundamental and therefore, since $\not{H} \neq \iota$, such that $\not{H}$ is not a congruence. In [11], it is shown that the varieties $\mathcal{V}\left(\mathcal{N}\left(Z_{p}, Z_{p}\right)\right), p$ a prime, and $\mathcal{V}(\mathcal{N}(Z, Z))$ are pairwise incomparable and that they are minimal in the lattice of varieties of inverse semigroups with respect to the property of containing inverse semigroups in which Ft is not a congruence. 
Our next goal is to show that the varieties $\mathcal{V}\left(\mathcal{N}\left(F \mathcal{U}_{X}, F \mathcal{U}_{X}\right)\right)$ where $\mathcal{U}$ is any variety of groups and $|X| \geq \aleph_{0}$ have similar minimality properties.

Notation 5.7. For any $x, y \in X$, let

$$
e(x, y)=x x^{-1} x^{-1} x y y^{-1} y^{-1} y\left(x^{-1} y y^{-1} x\right)\left(x y y^{-1} x^{-1}\right)\left(y^{-1} x x^{-1} y\right)\left(y x x^{-1} y^{-1}\right) \text {. }
$$

LemMA 5.8. For any group $G$ and any $x_{1}, \ldots, x_{n} \in S=\mathcal{N}(G, G), n \geq 2$, let $e=e\left(x_{1}, \ldots, x_{n}\right)$ be defined by

$$
e=\prod_{i, j} e\left(x_{i}, x_{j}\right)
$$

Then

$$
\left(e, e x_{i}\right) \in \nvdash, \quad \text { for all } i=1, \ldots, n \text {. }
$$

Proof. The claim is clearly true if the $x_{i}$ are all idempotents or if the $x_{i}$ all lie in the unit group $G$ or if any one of the $x_{i}$ is zero. We consider the remaining cases.

Case (i). For some $i, x_{i} \in \mathcal{M}^{0} \backslash E\left(\mathcal{M}^{0}\right)$. Then $x_{i} x_{i}^{-1} \neq x_{i}^{-1} x_{i}$ so that $x_{i} x_{i}^{-1} x_{i}^{-1} x_{i}$ $=0$. Hence $e=0$ and (16) holds.

Case (ii). For some $i, j, x_{i} \in G$, the unit group of $S, x_{i} \neq 1$ and $x_{j} \in E\left(\mathcal{M}^{0}\right)$. Let $x_{j}=(a, 1, a)$ then

$$
x_{i}^{-1} x_{j} x_{j}^{-1} x_{i}=x_{i}^{-1}(a, 1, a) x_{i}=\left(a x_{i}, 1, a x_{i}\right) .
$$

Since $a x_{i} \neq a$,

$$
\left(x_{i}^{-1} x_{j} x_{j}^{-1} x_{i}\right) x_{j} x_{j}^{-1}=0 .
$$

Hence $e=0$ and (16) holds.

Corollary 5.9. Let $G$ be a group, $\mathcal{V}=\mathcal{V}(\mathcal{N}(G, G))$ and $S \in \mathcal{V}$. For any $x_{1}, \ldots, x_{n} \in S$, with the notation of Lemma 5.8

$$
\left(e, e x_{i}\right) \in \not{H}, \quad \text { for all } i=1, \ldots, n \text {. }
$$

PROOF. The statement (17) is equivalent to the equations

$$
e=\left(e x_{i}\right)\left(e x_{i}\right)^{-1}=\left(e x_{i}\right)^{-1}\left(e x_{i}\right) \text {. }
$$

By Lemma 5.8, the equations (18) are identities for the variety $\mathcal{V}$ generated by $\mathcal{N}(G, G)$ and therefore hold in any member of $\mathcal{V}$, such as $S$. But this is equivalent to $(17)$.

THEOREM 5.10. Let $\mathcal{U}$ be a nontrivial variety of groups and $|X| \geq \aleph_{0}$. Then the variety $\mathcal{V}=\mathcal{V}\left(\mathcal{N}\left(F U_{X}, F U_{X}\right)\right)$ is minimal with respect to the properties

(i) $F \mathcal{V}_{X}$ is fundamental,

(ii) $\mathcal{V} \cap \mathcal{G}=\mathcal{U}$.

ProOF. Let $\mathcal{W}$ be a variety which satisfies (i) and (ii) and for which $\mathcal{W} \subseteq \mathcal{V}$. By Theorem 2.3 and Lemma $5.1, \mathcal{V}$ is a variety of completely semisimple semigroups and therefore so also is $\mathcal{W}$. Now it is not difficult to show that $\mathcal{N}\left(F U_{X}, F U_{X}\right)$ is embeddable in the product

$$
\prod_{n} \mathcal{N}\left(F_{n}(\mathcal{U}), F_{n}(\mathcal{U})\right)
$$


Therefore, in order to establish that $\mathcal{W}=\mathcal{V}$, it suffices to show that $\mathcal{N}\left(F_{n}(\mathcal{U}), F_{n}(\mathcal{U})\right)$ $\in \mathcal{W}$ for each $n$.

Let $|X|=\aleph_{0}, x_{1}, \ldots, x_{n} \in X$ and $F_{n}(I)$ be the inverse subsemigroup of $F I_{X}$ generated by $x_{1}, \ldots, x_{n}$. As it should not cause any confusion, let us denote the restrictions of $\rho_{\mathcal{u}}$ and $\rho_{\mathcal{W}}$ to $F_{n}(I)$ also by $\rho_{\mathcal{U}}$ and $\rho_{\mathcal{W}}$, respectively. Then $F_{n}(\mathcal{U})=$ $F_{n}(I) / \rho u$ and $F_{n}(\mathcal{W})=F_{n}(I) / \rho \mathcal{w}$. Let $s_{i}=x_{i} \rho \mathcal{w}, g_{i}=x_{i} \rho u$ and $e=e\left(s_{1}, \ldots, s_{n}\right)$ be defined as in Lemma 5.8. Then, by Corollary 5.9, the elements $e s_{i}$ are all $\not{k}$ equivalent to $e$. However, the mapping $\theta: s_{i} \rightarrow g_{i}(x=1, \ldots, n)$ determines an epimorphism $\theta$ of $F_{n}(\mathcal{W})$ onto $F_{n}(\mathcal{U})$. But the $e s_{i}$ all lie in the subgroup $H_{e}$ of $F_{n}(\mathcal{W})$ and generate a subgroup $H$, say. Therefore $\theta$ must map $H$ onto $F_{n}(\mathcal{U})$. However, $H \in \mathcal{W} \cap \mathcal{G}=\mathcal{U}$ and is generated by $n$ elements. Therefore, $\theta$ must induce an isomorphism of $H$ onto $F_{n}(\mathcal{U})$.

Goal. Our goal now is to show that $\mathcal{W}$ contains $\mathcal{N}(H, H)$.

By (i), $W=F W_{X}$ is fundamental. Hence, for every $a \in H^{\prime}=H \backslash\{e\}$, there exists an idempotent $e(a)<e$ such that

$$
a^{-1} e(a) a \neq e(a)
$$

Let $K_{a}$ be the inverse subsemigroup of $W$ generated by $e(a)$ and $H$. Let $J(e(a))$ be the principal ideal of $K_{a}$ generated by $e(a)$ and let $I(e(a))$ be the ideal of $K_{a}$ consisting of the elements of $J(e(a))$ which do not generate $J(e(a))$. Since $K_{a} \subseteq W \in \mathcal{W}, K_{a}$ is completely semisimple. Hence $K_{a} \backslash I(e(a))$ contains just the two $J$-classes $H$ and $J(e(a)) \backslash I(e(a))$. Let $L_{a}=K_{a} / I(e(a))$. Then $L_{a}$ must be an ideal extension of the Brandt semigroup $B_{a}=J(e(a)) / I(e(a))$ by the group $H$ with a zero adjoined.

We may regard $B_{a}$ as being the set $(J(e(a)) \backslash I(e(a))) \cup\{0\}$ where the multiplication is inherited from $W$ with the exception that if, for $u, v \in B_{a}$, either $u v \in I(e(a))$, in $W$, or $u=0$ or $v=0$, in $B_{a}$, then we put $u v=0$.

Now since $e(a)<e$, any idempotent of $B_{a}$ can be expressed in the form

$$
h_{1}^{-1} e(a) h_{1} h_{2}^{-2} e(a) h_{2} \cdots h_{n}^{-1} e(a) h_{n}, \quad h_{i} \in H .
$$

Since the product of distinct idempotents in $J(e(a))$ falls into $I(e(a))$, the nonzero idempotents of $B_{a}$ are of the form $h^{-1} e(a) h(h \in H)$, that is

$$
E\left(B_{a}\right) \backslash\{0\}=\left\{h^{-1} e(a) h: h \in H\right\} .
$$

In particular, $H$ is the unit group of $L_{a}$. Now let

$$
P=\prod_{a \in H^{\prime}} L_{a}
$$

and let

$$
Q=\left\{f \in P \text { : either (i) for all } a, f(a) \in H \text { or (ii) for all } a, f(a) \in B_{a}\right\} .
$$

Then $Q$ is an inverse subsemigroup of $P$ and

$$
R=\left\{f \in Q: f(a)=0, \text { for some } a \in H^{\prime}\right\}
$$

is an ideal of $Q$. The Rees quotient $S=Q / R$ is then the union of the unit group

$$
\prod_{a \in H^{\prime}} H
$$


and the Brandt semigroup

$$
B=\left(\prod_{a \in H^{\prime}} B_{a}\right) / R .
$$

We now embed $\mathcal{N}(H, H)$ in $S$.

Let $\varphi: \mathcal{N}(H, H) \rightarrow S$ be defined as follows: for all $a \in H^{\prime}$,

$$
\begin{aligned}
(g \varphi)(a) & =g, \quad g \in H, \\
((h, 1, k) \varphi)(a) & =h^{-1}(e(a)) k, \quad(h, 1, k) \in \mathcal{M}(H), \\
0 \varphi & =0 .
\end{aligned}
$$

Clearly $\varphi$ is one-to-one on $H$. Now let $r=(h, 1, k)$ and $s=\left(h^{\prime}, 1, k^{\prime}\right)$ be distinct nonzero elements of $\mathcal{M}(H)$. Then either $h \neq h^{\prime}$ or $k \neq k^{\prime}$. First consider the case $h \neq h^{\prime}$ and let $a=h^{\prime} h^{-1}(\neq e)$. Suppose that $(r \varphi)(a)=(s \varphi)(a)$. Then $h^{-1} e(a) k=\left(h^{\prime}\right)^{-1} e(a) k^{\prime}$ and, since $e(a)<e$, we have $e(a)=h h^{-1} e(a) k k^{-1}=$ $h\left(h^{\prime}\right)^{-1} e(a) k^{\prime} k^{-1}$. Hence

$$
e(a)=e(a) e(a)^{-1}=h\left(h^{\prime}\right)^{-1} e(a)\left(h\left(h^{\prime}\right)^{-1}\right)^{-1}=a^{-1} e(a) a,
$$

contradicting (19). Therefore, $(r \varphi)(a) \neq(s \varphi)(a)$ so that $r \varphi \neq s \varphi$. We obtain the same conclusion if $k \neq k^{\prime}$. Thus $\varphi$ is one-to-one on $\mathcal{M}(H)$ and, therefore, on the whole of $\mathcal{N}(H, H)$.

It is easily seen that $\varphi$ is a homomorphism on $H$. Now let $r$ and $s$ be as above. First suppose that $k=h^{\prime}$. Then $r s=\left(h, 1, k^{\prime}\right)$ and, for all $a \in H^{\prime},((r \varphi)(s \varphi))(a)=$ $h^{-1} e(a) k\left(h^{\prime}\right)^{-1} e(a) k^{\prime}=h e(a) k^{\prime}=((r s) \varphi)(a)$ so that $(r \varphi)(s \varphi)=(r s) \varphi$.

Now suppose that $k \neq h^{\prime}$. Then $(r s) \varphi=0 \varphi=0$. On the other hand,

$$
r^{-1} r=(k, 1, k) \text { and } s s^{-1}=\left(h^{\prime}, 1, h^{\prime}\right)
$$

so that $\left(r^{-1} r\right) \varphi$ and $\left(s s^{-1}\right) \varphi$ are distinct elements of $B$. Since $\left(r^{-1} r\right) \varphi$ and $\left(s s^{-1}\right) \varphi$ are clearly idempotents as well, their product in the Brandt semigroup $B$ must be zero. Thus, for some $a \in H^{\prime}$

$$
0=\left(\left(r^{-1} r\right) \varphi\left(s s^{-1}\right) \varphi\right)(a)=k^{-1} e(a) k\left(h^{\prime}\right)^{-1} e(a) h^{\prime} .
$$

Then

$$
\begin{aligned}
((r \varphi)(s \varphi))(a) & =h^{-1} e(a) k\left(h^{\prime}\right)^{-1} e(a) k^{\prime} \\
& =h^{-1} k\left(k^{-1} e(a) k\right)\left(h^{\prime}\right)^{-1} e(a) h^{\prime}\left(h^{\prime}\right)^{-1} k^{\prime} \\
& =0 .
\end{aligned}
$$

Thus $(r \varphi)(s \varphi)=0=(r s) \varphi$ so that $\varphi$ is a homomorphism on $\mathcal{M}(H)$.

Now let $g \in H$ and $r$ be as before. For all $a \in H^{\prime},((g r) \varphi)(a)=\left(h g^{-1}, 1, k\right) \varphi(a)$ $=g h^{-1} e(a) k=(g \varphi)(r \varphi)(a)$ so that $(g r) \varphi=(g \varphi)(r \varphi)$. Similarly $(r g) \varphi=(r \varphi)(g \varphi)$. Clearly $(0 x) \varphi=(x 0) \varphi=(0 \varphi)(x \varphi)=(x \varphi)(0 \varphi)=0$, for all $x \in \mathcal{N}(H, H)$, so that $\varphi$ is a homomorphism and therefore a monomorphism of $\mathcal{N}(H, H)$ into $S$. Since $S \in \mathcal{W}$, it follows that $\mathcal{N}(H, H) \in \mathcal{W}$, as required. This completes the proof of Theorem 5.10.

6. The interval $\left[C, A G^{\max }\right]$. In Corollary 3.8 , we saw that for any variety $\mathcal{V}$ such that $C \subseteq \mathcal{V} \subseteq A G^{\max }, F \mathcal{V}_{X}$ is combinatorial, completely semisimple and $E$-unitary. We can now say something about the size of this interval.

Recall that, for any prime $p, \mathcal{V}_{p}$ is the variety of inverse semigroups generated by $\mathcal{N}\left(Z_{p}, Z_{p}\right)$. 
LeMma 6.1 (SEE $[\mathbf{1 1}]$ ). (i) For any positive integer $p \geq 2, V_{p}$ satisfies the identity

$$
\left(x^{p}\right)^{2}=x^{p} .
$$

(ii) The variety $C$ satisfies the identities

$$
y y^{-1} x^{n} y y^{-1}=y y^{-1}\left(x y y^{-1}\right)^{n} \quad \text { for } n=1,2,3, \ldots
$$

LEMMA 6.2. For any distinct primes $p, q$, the identity

$$
y y^{-1} x^{p q} y y^{-1}=y y^{-1}\left(x^{p} y y^{-1}\right)^{q}
$$

is satisfied by $\nu_{p}$ but not by $\nu_{q}$.

Proof. Consider any elements $x, y \in \mathcal{N}\left(Z_{p}, Z_{p}\right)$. If $x \in Z_{p}$, an additive group, then $p x=0$ and equality holds in (21). If $x$ is an idempotent in $\mathcal{M}\left(Z_{p}\right)$ then it is clear that equality again holds. If $x \in \mathcal{M}\left(Z_{p}\right)$ but is not an idempotent, then $x^{2}=0$ so that both sides of (21) are zero and equality again holds.

To see that $\mathcal{N}\left(Z_{q}, Z_{q}\right)$, and therefore also $\mathcal{V}_{q}$, does not satisfy (21), let $x=1 \in Z_{q}$, the unit group, and $y=(0,1,0)$. Then the left-hand side of $(21)$ is - noting that 0 is the identity of $Z_{q}-$

$$
(0,1,0) \cdot 0 \cdot(0,1,0)=(0,1,0)
$$

while the right-hand side is

$$
\begin{aligned}
(0,1,0) \cdot(p \cdot(0,1,0))^{q} & =(0,1,0) \cdot(q-p, 1,0)^{q} \\
& =\text { the zero in } \mathcal{N}\left(Z_{q}, Z_{q}\right)
\end{aligned}
$$

since $(q-p, 1,0)^{2}$ is zero in $\mathcal{N}\left(Z_{q}, Z_{q}\right)$. Thus (21) does not hold in $\mathcal{V}_{q}$.

Proposition 6.3. The varieties $\nu_{p} \vee C, p$ is a prime, constitute a countably infinite family of distinct varieties in $\left[C, A G^{\max }\right]$.

ProOF. Since $\mathcal{V}\left(Z_{p}\right) \subseteq A G$, for all primes $p$, it follows from Proposition 5.2 (iii) that $V_{p} \vee C \subseteq A G^{\max }$, so that, for all primes $p, \nu_{p} \vee C \in\left[C, A G^{\max }\right]$.

Now let $p$ and $q$ be distinct primes. By Lemma 6.1(ii), $C$ satisfies the identity (21) as does $\nu_{p}$, by Lemma 6.2. Therefore $\mathcal{V}_{p} \vee C$ also satisfies (21). However, by Lemma 6.2, $\mathcal{V}_{q} \vee C$ does not satisfy (21). Hence $\mathcal{V}_{p} \vee C$ and $\mathcal{V}_{q} \vee C$ are distinct.

7. The varieties $\left[x^{n} x^{-n}=x^{-n} x^{n}\right]$. For each positive integer $n$, let $\mathcal{W}_{n}$ denote the variety defined by the identity $x^{n} x^{-n}=x^{-n} x^{n}$. By virtue of Lemma 2.3, the varieties $\mathcal{W}_{n}$ have a significant role to play in the study of the lattice of varieties of inverse semigroups. We apply the techniques of earlier sections in order to obtain information regarding the relatively free objects in $\mathcal{W}_{n}$. In doing so, we see that there are fundamental relatively free objects which are not combinatorial.

THEOREM 7.1. Let $n \geq 2, \mathcal{W}=\mathcal{W}_{n}$ and $|X| \geq \aleph_{0}$. Then $F \mathcal{W}_{X}$ is fundamental and E-unitary but not combinatorial.

Proof. Since any group satisfies the identity $x^{n} x^{-n}=x^{-n} x^{n}$, it follows that $\mathcal{G} \subseteq \mathcal{W}$. hence, $\operatorname{ker} \rho_{\mathcal{W}}=\operatorname{ker} \rho_{\mathcal{G}}=E\left(F I_{X}\right)$ so that $F \mathcal{W}_{X}$ is $E$-unitary.

Now let $\mathcal{V}=\mathcal{V}\left(\mathcal{N}\left(F \mathcal{G}_{X}, F \mathcal{G}_{X}\right)\right)$. By Lemma 5.1, $\mathcal{V} \subseteq \mathcal{W}$. Now $F \mathcal{G}_{X}$ is a subgroup of $\mathcal{N}\left(F \mathcal{G}_{X}, F \mathcal{G}_{X}\right)$, so that $F \mathcal{G}_{X} \in \mathcal{V}$ and $\mathcal{G} \subseteq \mathcal{V}$. Hence $\operatorname{ker} \rho \mathcal{v}=$ 
$\operatorname{ker} \rho_{\mathcal{G}}=\operatorname{ker} \rho_{\mathcal{W}}$. Since $\mathcal{N}\left(F \mathcal{G}_{X}, F \mathcal{G}_{X}\right)$ is fundamental, it follows from Theorem 3.1, that $F \mathcal{V}_{X}$ is fundamental and therefore, by Theorem 3.3, that $F \mathcal{W}_{X}$ is fundamental.

To see that $F \mathcal{W}_{X}$ is not combinatorial, let $y \in X$ and $a=y \rho_{\mathcal{W}}$. By the defining identity for $\mathcal{W}, a^{n} a^{-n}=a^{-n} a^{n}$ so that $a^{n}$ lies in a subgroup of $F \mathcal{W}_{X}$. However, the mapping $x \rho \mathcal{W} \rightarrow x \rho_{\mathcal{G}}$ defines a homomorphism of $F \mathcal{W}_{X}$ onto $F \mathcal{G}_{X}$ in which $y \rho \mathcal{W}$ is mapped onto a free generator. Hence, $\left(y \rho_{\mathcal{G}}\right)^{n}$ is not an idempotent. Therefore $a^{n}=(y \rho \mathcal{W})^{n}$ is not an idempotent and the $\mathscr{H}$-class of $a^{n}$ is a nontrivial group.

\section{REFERENCES}

1. A. H. Clifford and G. B. Preston, The algebraic theory of semigroups, Math. Surveys, no. 7, Amer. math. Soc., Providence, R.I., 1961.

2. L. M. Gluskin, Inverse semigroups, Zap. Meh.-Mat. Fak. i Har'kov. Mat. Obšc. (4) 28 (1961), 103-110 (Russian).

3. J. M. Howie, An introduction to semigroup theory, Academic Press, London, 1976.

4. F. Pastijn, Inverse semigroup varieites generated by E-unitary inverse semigroups, Semigroup Forum 24 (1982), 87-88.

5. F. Pastijn and P. G. Trotter, Lattices of completely regular semigroup varieties, Pacific J. Math. 119 (1985), 191-214.

6. M. Petrich, Inverse semigroups, Wiley, New York, 1984.

7. M. Petrich and N. R. Reilly, E-unitary covers and varieties of inverse semigroups, Acta Sci. Math. (Szeged) 46 (1983), 59-72.

8. N. R. Reilly, Free generators in free inverse semigroups, Bull. Austral. Math. Soc. 7 (1972), 407-424.

9. ___ Varieties of completely semisimple inverse semigroups, J. Algebra 65 (1980), 427444.

10. __ Free inverse semigroups, Jozsef Attila Univ. Szeged Colloq. Math. Soc. János Bolyai 20, North-Holland, Amsterdam, 1979.

11. __ Minimal non-cryptic varieties of inverse semigroups, Quart. J. Math. Oxford (2) (to appear).

12. H. E. Scheiblich, Free inverse semigroups, Proc. Amer. Math. Soc. 38 (1973), 1-7.

Department of Mathematics, Simon Fraser University, Burnaby, British COlumbia, CANada V5A 1S6

Department of Mathematics, The University of Tasmania, Hobart, tasmaNia, Australia 7001 